\title{
Predictive Analytic Model for Diagnosis of Ectopic Pregnancy
}

\author{
Ploywarong Rueangket* and Kristsanamon Rittiluechai \\ Department of Obstetrics and Gynecology, Phramongkutklao Hospital, Bangkok, Thailand
}

Objective: Ectopic pregnancy (EP) is a serious condition. Delayed diagnosis could lead to life-threatening outcomes. The study aimed to develop a diagnostic predictive model for EP to approach suspected cases with prompt intervention before the rupture occurred.

Methods: A retrospective cross-sectional study enrolled 347 pregnant women presenting first-trimester complications (abdominal pain or vaginal bleeding) with diagnosis suspected of pregnancy of unknown location, who were eligible and underwent chart review. The data including clinical risk factors, signs and symptoms, serum human chorionic gonadotropin (hCG), and ultrasound findings were analyzed. The statistical predictive score was developed by performing logistic regression analysis. The testing data of 30 patients were performed to test the validation of predictive scoring.

OPEN ACCESS

Edited by: Christine Wyns,

Catholic University of Louvain, Belgium

Reviewed by: Salim Alfred Bassil, Al-Arz Hospital, Lebanon Mohd Faizal Ahmad, National University of

Malaysia, Malaysia

*Correspondence:

Ploywarong Rueangket ploywarong.24@gmail.com

Specialty section

This article was submitted to Obstetrics and Gynecology,

a section of the journal

Frontiers in Medicine

Received: 25 December 2020

Accepted: 26 February 2021

Published: 29 April 2021

Citation:

Rueangket $P$ and Rittiluechai $K$ (2021)

Predictive Analytic Model for

Diagnosis of Ectopic Pregnancy.

Front. Med. 8:646258.

doi: 10.3389/fmed.2021.646258
Results: From a total of 22 factors, logistic regression method-derived scoring model was based on five potent factors (history of pelvic inflammatory disease, current use of emergency pills, cervical motion tenderness, serum $\mathrm{hCG} \geq 1,000 \mathrm{mlU} / \mathrm{ml}$, and ultrasound finding of adnexal mass) using a cutoff score $\geq 3$. This predictive index score was able to determine ectopic pregnancy with an accuracy of $77.8 \%$ [95\% confidence interval $(\mathrm{Cl})=$ 73.1-82.1], specificity of $91.0 \%(95 \% \mathrm{Cl}=62.1-72.0)$, sensitivity of $67.0 \%(95 \% \mathrm{Cl}=$ 88.0-94.0), and area under the curve of 0.906 (95\% $\mathrm{Cl}=0.875-0.937)$. In the validation group, no patient with negative result of this score had an EP.

Conclusion: Statistical predictive score was derived with high accuracy and applicable performance for EP diagnosis. This score could be used to support clinical decision making in routine practice for management of EP.

Keywords: ectopic pregnancy, first trimester complications, predictive scoring model, pregnancy of unknown location, serum human chorionic gonadotropin, statistical model

\section{INTRODUCTION}

Ectopic pregnancy $(\mathrm{EP})$ is a potentially life-threatening complication when treatment is delayed. Reported maternal death by Confidential Enquiry into maternal deaths (CEMD) in the United Kingdom researcher as the fourth leading cause of death (1). Despite a comprehensive medical evaluation including patient risk assessment, clinical evaluation, and investigations, the rate of deaths in the United Kingdom has not declined since 1991. In fact, one-third of women with EP have no clinical signs, and up to $10 \%$ are asymptomatic $(2,3)$. The use of transvaginal ultrasonography (TVS) was found to improve the accuracy of diagnosis. However, only $73.9 \%$ of tubal EPs were visualized by initial TVS (4). 
Until recently, serial measurements of serum human chorionic gonadotropin (hCG) levels had been shown to benefit EP detection. Although with many research data, the serum hCG's patterns were still unable to differentiate those with EP from intrauterine pregnancy or abortion precisely enough (5). Even though the longer follow-up serum hCG may help to locate gestation, $32 \%$ of ruptured EP usually would occur within the first $48 \mathrm{~h}$, which is the most critical timing for physicians to offer the intervention before the rupture. Unfortunately, the uncertainty remained in the initial diagnosis leading to high mortality and infertility risk for the next pregnancy (6).

Attempts to develop a predictive model began in the early 20th century for early diagnosis patients with EP by using clinical data, serum marker (hCG and/or progesterone), and ultrasound finding. One widely known statistical model using serum hCG to predict EP is the M1 and M4 model by Condous et al. (7). Whereas, the first M1 model seems to have good sensitivity and specificity, the positive predictive value is still low. Later, the M4 model, based on serial serum hCG values $48 \mathrm{~h}$ apart, demonstrated a better predictive ability but lower performance in different populations (8).

The challenge in identifying the EP among patients presenting early pregnancy complications is crucial, because early detection could reduce maternal morbidity and mortality and also preserved future fertility. Despite much data and research, no infallible EP predictive model is available. This research aimed to create an effective predictive model to diagnose EP and extract the association factors inside the data.

\section{MATERIALS AND METHODS}

\section{Study Designs and Subjects}

The cross-sectional study was reviewed and approved by the Royal Thai Army Medical Department Institutional Review Board before conducting the study using electronic medical records of Phramongkutklao Hospital between October 2010 and April 2020, involving women presenting vaginal bleeding and/or abdominal pain during the first trimester of pregnancy. All patients underwent transvaginal ultrasound. Inclusion criteria comprised patients who had been evaluated and diagnosed with pregnancy of unknown location (PUL), pregnancy with no signs of intrauterine pregnancy, or an extrauterine pregnancy via ultrasonography at the first visit. Patients presenting signs clinically suggestive of ruptured EP (clinical instability or sign of intra- abdominal hemorrhage) or who show any evidence of intrauterine gestational content or EP (adnexal mass consisted of fetal pole or fetal heart motion) by ultrasound at the first visit were excluded.

The primary outcome of interest was the final diagnosis of EP or non-EP. The diagnosis of EP was confirmed by postoperative pathological result and abnormality in pattern measurement of serial hCG levels (plateau or abnormal increased/decreased patterns) with unidentified chorionic villi in uterine cavity or having undergone treatment with medication. In the non-EP group, final diagnosis included threatened abortion, spontaneous

Abbreviations: EP, ectopic pregnancy; PUL, pregnancy of unknown location. abortion, or miscarriage. They needed to be confirmed by pathological result or subsequent decrease of serum hCG level in miscarriage pattern or continuing intrauterine pregnancy by subsequent ultrasound finding.

Demographic data, history of risk factors, clinical manifestations, initial serum hCG levels, and ultrasound results were reviewed and recorded, totaling 22 factors. All factors were extracted and selected from literature reviews to obtain statistical significance relevant to our research outcome.

\section{Statistical Analysis}

The method used for EP prediction was logistic regression. Collected data were checked, coded, and then analyzed using STATA 15.1/IC (StataCorp). The data were chronologically split up in a training and a test set of 9:1 ratio. In a training dataset, frequency distribution of demographic characteristics and factors were calculated to determine descriptive statistics of the samples. Binary logistic regression analysis was used to determine the risk factors associated with EP. The magnitude of association was presented as crude odds ratios (ORs) with 95\% confidence interval (CI). A $p<0.05$ was considered as statistically significant. Differences identified between subgroups of patients are considered hypothesis-generating and require confirmation in independent studies. Multivariate analysis was performed to adjust confounders by using logistic regression analysis with enter method, which is a default function of STATA Software to simultaneously eliminate (backward stepwise elimination) independent factors in the model. All statistically significant predictive factors in multivariate analysis were used to create score. The probability of EP diagnosed with each score value was calculated, and then sensitivity, specificity, and accuracy of various score cutoff were computed. Maximum likelihood estimation was used to obtain coefficients from logistic regression analysis. The score was applied to the testing dataset for validation. Regression coefficient- based scoring algorithms were performed, and scoring system for EP diagnostic model was derived.

\section{RESULTS}

\section{Characteristics of Study Populations}

From a total of 1,275 pregnant women presenting at early pregnancy with complications, 347 (27\%) pregnant women with suspected PUL at initial diagnosis were identified. The mean age was 30 years with $43.8 \%$ nullipara. In 347 patients with PUL, $55 \%(n=191)$ were EP, and $45 \%(n=156)$ were non-EP. Among 156 patients with non-EP, $20(12.8 \%)$ were threatened abortion, one $(0.6 \%)$ blighted ovum, one $(0.6 \%)$ corpus luteal leakage, and other 134 (85.9\%) were spontaneous abortion. The majority of patients with EP had no underlying disease (88.5\%), no history of pelvic surgery (79.6\%), no previous EP (95.3\%), and no history of pelvic inflammatory disease (PID) (90.6\%). There was no statistically significant difference in gestational age (GA) at diagnosis between EP patients (52.6 \pm 16.1 days) and non-EP patients (50.7 \pm 15.8 days). Ninety-seven percent of patients with EP were non-smoker, and only $16.6 \%$ were in 
TABLE 1| Demographic characteristics of the study population.

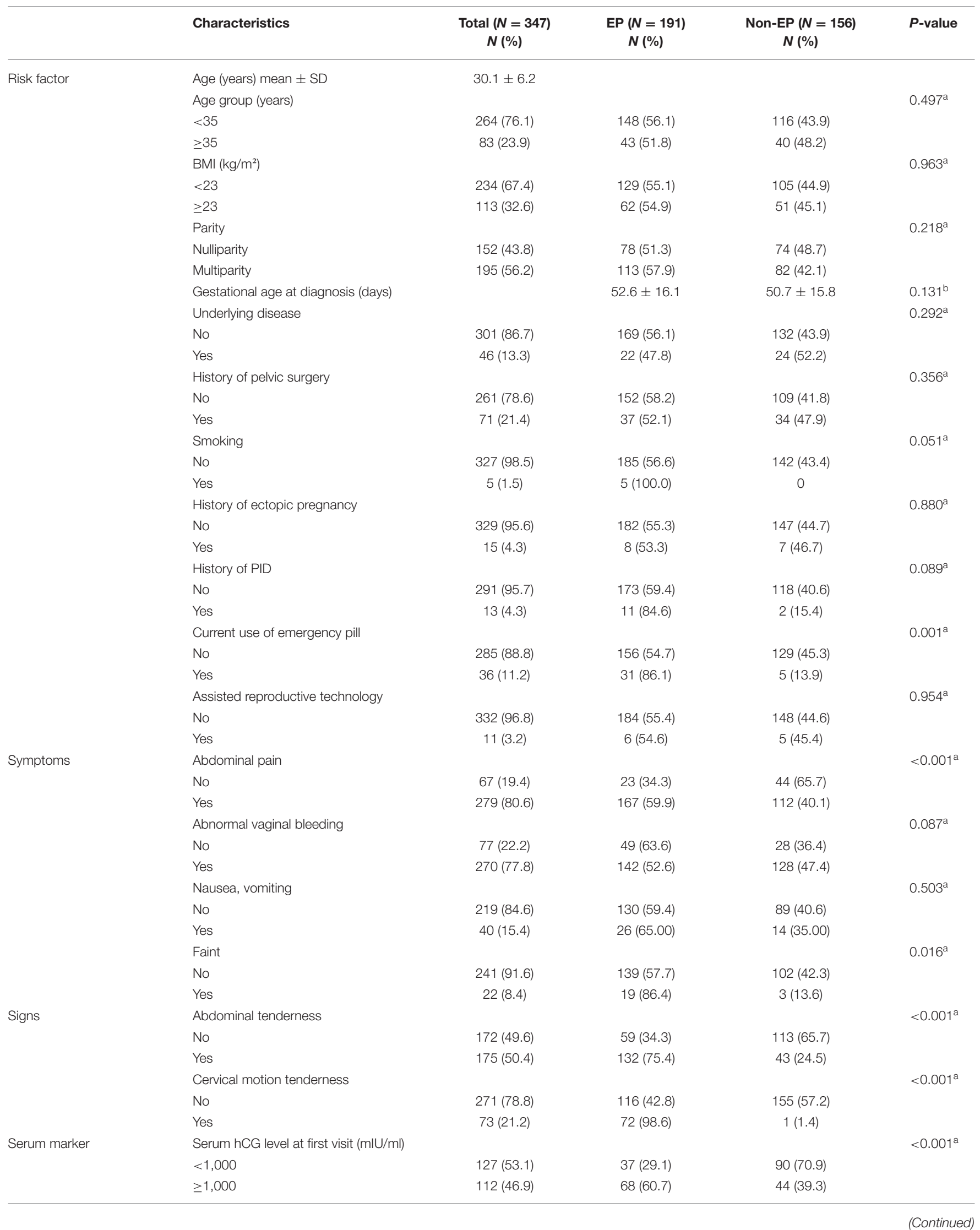


TABLE 1 | Continued

\begin{tabular}{|c|c|c|c|c|c|}
\hline & Characteristics & $\begin{array}{c}\text { Total }(n=347) \\
n(\%)\end{array}$ & $\begin{array}{c}\mathrm{EP}(n=191) \\
n(\%)\end{array}$ & $\begin{array}{c}\text { Non-EP }(n=156) \\
n(\%)\end{array}$ & $P$-value \\
\hline \multirow[t]{7}{*}{ Ultrasound findings } & Intra-uterine anechoic content & & & & $0.908^{\mathrm{a}}$ \\
\hline & Yes & $36(10.5)$ & $20(55.6)$ & $16(44.4)$ & \\
\hline & Endometrial thickness $>14 \mathrm{~mm}$ & & & & $0.461^{a}$ \\
\hline & $\begin{array}{l}\text { Adnexal mass of complex } \\
\text { echogenicity }\end{array}$ & & & & $<0.001^{a}$ \\
\hline & No & $152(43.4)$ & $23(15.1)$ & $129(84.9)$ & \\
\hline & Yes & $194(56.1)$ & $167(86.1)$ & 27 (13.9) & \\
\hline & Free fluid in cul-de-sac & & & & $<0.001^{\mathrm{a}}$ \\
\hline
\end{tabular}

SD, standard deviation; BMI, body mass index; PID, pelvic inflammatory disease.

${ }^{a} \chi^{2}$ Test.

bt Test.

TABLE 2 | Univariate and multivariate logistic regression analyses of the factors significantly associated with ectopic pregnancy and scoring system.

\begin{tabular}{|c|c|c|c|c|c|c|c|}
\hline $\begin{array}{l}\text { Features } \\
\text { total case }(N)=347\end{array}$ & Missing data (\%) & \multicolumn{2}{|c|}{ Univariate analysis } & \multicolumn{3}{|c|}{ Multiple analysis } & Risk score (13) \\
\hline History of PID & $43(12.3 \%)$ & $\begin{array}{c}3.75 \\
(0.82-17.23)\end{array}$ & 0.089 & $\begin{array}{c}7.30 \\
(0.63-85.08)\end{array}$ & 0.112 & 1.99 & 2 \\
\hline $\begin{array}{l}\text { History of ectopic } \\
\text { pregnancy }\end{array}$ & $3(0.86 \%)$ & $\begin{array}{c}0.92 \\
(0.33-2.60)\end{array}$ & 0.880 & & & & \\
\hline Abdominal pain & $1(0.3 \%)$ & $\begin{array}{c}2.85 \\
(1.63-4.98)\end{array}$ & $<0.001$ & & & & \\
\hline Faint & $84(24.2 \%)$ & $\begin{array}{c}4.65 \\
(1.34-16.13)\end{array}$ & 0.016 & & & & \\
\hline Abdominal tenderness & - & $\begin{array}{c}5.88 \\
(3.69-9.37)\end{array}$ & $<0.001$ & & & & \\
\hline $\begin{array}{l}\text { Serum hCG level at first } \\
\text { visit }(\mathrm{ml} / \mathrm{ml}) \geq 1,000\end{array}$ & 108 (31.1\%) & $\begin{array}{c}3.76 \\
(2.19-6.44)\end{array}$ & $<0.001$ & $\begin{array}{c}2.63 \\
(1.27-5.46)\end{array}$ & 0.009 & 0.96 & 1 \\
\hline $\begin{array}{l}\text { Adnexal mass of } \\
\text { complex echogenicity }\end{array}$ & $1(0.3 \%)$ & $\begin{array}{c}34.69 \\
(19.01-63.32)\end{array}$ & $<0.001$ & $\begin{array}{c}9.83 \\
(1.20-80.73)\end{array}$ & 0.033 & 2.29 & 2.5 \\
\hline Free fluid in cul-de-sac & $6(1.7 \%)$ & $\begin{array}{c}8.42 \\
(5.05-14.05)\end{array}$ & $<0.001$ & & & & \\
\hline
\end{tabular}

ORs, odds ratios; Cl, confidence interval; PID, pelvic inflammatory disease.

current use of emergency pill. Regarding clinical presentation and assessment, $87.4 \%$ of patients in the EP group had abdominal pain, and $74.3 \%$ of them had noticed abnormal vaginal bleeding. There was abdominal tenderness in $69.1 \%$ of patients with EP, and $37.7 \%$ had cervical motion tenderness. On the other side, $35.6 \%$ (68 of 191) of patients with EP presented with serum hCG $\geq 1,000$, whereas $45 \%$ of patients with EP did not obtain blood examination. When compared with patients with no EP, complex adnexal mass and free fluid in cul-de-sac by initial ultrasound finding were more common in EP patients, which was 87.4 vs. $17.3 \%$ and 62.3 vs. $17.3 \%$, respectively, as presented in Table 1.

Using logistic regression in the training dataset, from a total of 22 factors, the results highlighted eight significant factors in univariate analysis, exhibiting an established relationship with EP, including current use of emergency pill, abdominal pain, fainting, 
TABLE 3 | Predictive ectopic pregnancy scoring model, with the acronym APrllE.

\begin{tabular}{|c|c|c|c|c|c|}
\hline Risk factor & Adnexal mass of complex echogenicity & Pain related to cervix & Infection of pelvis & $\geq 1,000$ of serum $\mathrm{hCG}$ & Emergency pill current use \\
\hline
\end{tabular}

TABLE 4 | Predictive scoring system cutoff detailed report performance.

\begin{tabular}{llllll}
\hline \multicolumn{7}{c}{ Detailed report of sensitivity and specificity } \\
\hline Cutoff & Sensitivity & Specificity & $\begin{array}{c}\text { Corrected } \\
\text { classified }\end{array}$ & LR+ & LR- \\
& & & & & \\
\hline$\geq 1$ & $94.8 \%$ & $58.3 \%$ & $78.4 \%$ & 2.27 & 0.09 \\
$\geq 1.5$ & $91.1 \%$ & $79.5 \%$ & $85.9 \%$ & 4.44 & 0.11 \\
$\geq 2$ & $90.1 \%$ & $81.4 \%$ & $86.2 \%$ & 4.84 & 0.12 \\
$\geq 2.5$ & $89.5 \%$ & $82.1 \%$ & $86.2 \%$ & 4.99 & 0.13 \\
$\geq 3$ & $67.0 \%$ & $91.0 \%$ & $77.8 \%$ & 7.47 & 0.36 \\
$\geq 3.5$ & $65.1 \%$ & $91.0 \%$ & $77.2 \%$ & 7.35 & 0.37 \\
$\geq 4$ & $46.1 \%$ & $98.1 \%$ & $69.5 \%$ & 23.96 & 0.55 \\
$\geq 4.5$ & $41.4 \%$ & $99.4 \%$ & $67.4 \%$ & 64.52 & 0.59 \\
\hline
\end{tabular}

$L R+$, positive likelihood ratio; $L R-$, negative likelihood ratio.

abdominal tenderness, cervical motion tenderness, initial serum hCG level $\geq 1,000 \mathrm{mIU} / \mathrm{ml}$, ultrasound findings of complex echogenicity, and finding of free fluid in cul-de-sac. Although history of PID and previous EP were not found to be statistically significant factors in our univariate analysis, these factors were proven to be clinically significant as risk factors of EP from many literature reviews (9-12). Thus, we selected history of PID and previous EP to evaluate in multivariate analysis. Stepwise method using backward elimination for factor selection was used in multivariate analysis, based on predictors and significant $p<0.2$. We found that the variables history of PID, current use of emergency pill, cervical motion tenderness, initial serum hCG level $\geq 1,000 \mathrm{mIU} / \mathrm{ml}$, and ultrasound findings of complex echogenicity were statistically significant factors to predict EP, as shown in Table 2.

The score of each five significantly predictive factors was adjusted according to the coefficient-based scoring ratio in multiple logistic regression, as revealed in Table 2. The statistical model was developed as a predictive scoring model with the acronym APrIlE score, as presented in Table 3. Thus, a predictive index score with cutoff level $\geq 3$ provided prediction of patients with EP with the best specificity of $91.0 \%$ (95\% CI $=62.1$ $72.0)$, with a good balance in sensitivity of $67 \%(95 \% \mathrm{CI}=$ 88.0-94.0). The area under the curve (AUC) was 0.906 (95\% CI $=0.875-0.937)$, as presented in Table 4. Lastly, the model was validated in a new group of patients with PUL $(n=30)$. We found that the predictive index score with cutoff $\geq 3$ could provide an exceptional performance of $90 \%(95 \% \mathrm{CI}=73.5-$ $97.9)$ in accuracy, specificity of $100 \%(95 \% \mathrm{CI}=83.9-100)$, and sensitivity of $66.7 \%$ (95\% CI $=29.9-92.5)$, with AUC of 0.905 ( $95 \% \mathrm{CI}=0.875-0.937)$. Among patients who were classified by the score in the low-risk group of EPs, none had been diagnosed with EP.

\section{DISCUSSION}

Overall, the data of 347 patients were analyzed by using univariate analysis, and 22 factors were studied. From a total 1,275 pregnant patients presenting first-trimester complications, 27.2\% were diagnosed with PUL. The incidence was similar to many studies, ranging between 7 and $31 \%$ (14-17). In our study, EP was diagnosed in $55 \%$ of PUL, which was similar to the $43 \%$ rate in one large prospective observational published study by Malek-Mellouli et al. (18). However, another retrospective study reported the wide range of 7 to $20 \%$ (1519), although a limitation of retrospective studies was that the complete definition to diagnose spontaneous resolution of EP or non-EP in PUL might be failed, as the true location of gestation was never known. In our study, we used the pathological report with unidentified chorionic villi in uterine cavity and the normal decline in serum hCG patterns to confirm miscarriage diagnosis.

The age of 35 years appears to be a risk factor of EP stated by the American College of Obstetricians and Gynecologists (ACOG) in 2018 (20). However, our study found that there was no statistically significant difference in EP and non-EP between the age groups $<35$ and $\geq 35$ years. This finding was similar to the multicenter case-control study by Cheng $\mathrm{Li}$ et al., who reported that age group was not a significant risk factor for EP (12). On the other hand, it appears that the mean age of our study population was $30.1 \pm 6.2$ years. The study by Essa et al. (21), also revealed that the majority of patients were in their 30s. Also, these findings match those of another large case-control study by Bouyer et al. (22), which found that $51.6 \%$ of patients was 30 years old. So far, we only believe that the age at 30 s could be a piece of a jigsaw puzzle that needed the matching pieces before the EP puzzle could be solved. As in the complex data to predict a single disease, only one factor could not be used to solve the problem. Another interesting finding was the GA at diagnosis. The mean GA at diagnosis of EP patients was 52.6 \pm 16.1 days $(7.5 \pm 2.3$ weeks $)$ and $50.7 \pm 15.8$ days $(7.2 \pm 2.2$ weeks) in non-EP patients. These results share similarities with a study by Saxon et al., in which the mean GAs at diagnosis of an unruptured tube and of those with a ruptured tube were $6.9 \pm 1.9$ and $7.2 \pm 2.2$ weeks, respectively (23). Despite that our study could not identify the differences in GA at diagnosis between the EP and non-EP groups, the systematic review study revealed that the first presenting symptoms of miscarriage most likely occurred around 5 weeks and 8 to 10 weeks of GA (24).

Theoretically, any condition that delays or interferes with the passage of an embryo through the fallopian tubes may increase the risk of EP (25). Despite the study by Berek et al., (26), which explained that up to one-half of EP cases have unidentified risk factors, a history of PID has a strong association with EP in 
our study. Also, from many studies (9-12), PID was also found to be one of the traditional risk factors and could be explained by its pathophysiology causing inflammation and disruption of tubal motility. Emergency contraceptive pills are another factor that could impair motility of fallopian tube. However, the use of emergency pills related to EP is still inconclusive from various studies (12, 27-29). In the result, using a different population, the treatment failure of emergency contraceptive pills was found to be related to EP. According to a history of previous EP in this study, there were only $4 \%$ of the patients in each study group who had a history of previous EP and shown insignificance relevant to EP. Although a case-control study reported that a history of previous EP was the strongest risk factor associated with EP (OR $=17.16,95 \% \mathrm{CI}=1.89-155.67)$ (29), other studies could not demonstrate the association between previous $\mathrm{EP}$ and subsequent EP $(18,21)$.

Several studies have found the association between cigarette smoking and EP $(22,29,30)$. Despite the fact that pathophysiology of smoking related to EP remains unclear, inhalation of cigarette smoking has shown the effect on the function of cilia and smooth muscles of fallopian tube presented by animal models (25). In this study, we found that only five patients were smokers, and all of them were diagnosed with EP. However, there was no significant difference between the two groups.

Many studies attempted to address clinical symptoms associated with EP. As noted by Malek-Mellouli et al. (18), abdominal pain with vaginal bleeding during the first trimester was strongly associated with EP. In addition to the study of Buckley et al. (31), the developed clinical prediction model for EP showed that cervical motion and abdominal tenderness were addressed as high and moderate risk factors for EP, respectively. Similarly, we found that abdominal pain, vaginal bleeding, cervical motion, and abdominal tenderness were shown to be the potent risk factors. However, we acknowledged that fainting was more relevant to EP than abdominal tenderness ( 86 vs. $60 \%$ ) in comparison with non-EP. However, 10 to $30 \%$ of patients with EP usually presented with unspecific clinical signs and symptoms $(2,26)$.

In patients with inconclusive symptom, ultrasound findings became an important investigation for diagnostic EP. Inhomogeneous adnexal mass separated from ovary and free fluid in cul-de-sac are important findings that could be related to EP. These two factors have sensitivity of 84 and $47.2 \%$ and specificity of 99 and $92.3 \%$ for EP diagnosed, respectively $(32,33)$. Also shown in this study, ultrasound findings of complex adnexal mass and free fluid were strong predictive factors for EP. Moreover, other findings such as endometrial thickness $>14 \mathrm{~mm}$ (sensitivity $48 \%$, specificity $66 \%$ ) and pseudo-gestational sac and empty uterus were found to be related to EP in some studies, as well $(32,33)$.

To date, serum hCG became another important aiding tool for EP diagnosis and management. Many published studies focused on the concept of discriminatory zone, which was the lowest level of serum hCG that should be detected in intrauterine gestational sac by ultrasound. The absence of intrauterine gestational structure sonographic signs when serum hCG increased above the discriminatory threshold was considered diagnostic of nonviable pregnancy or EP (34). With the availability of highresolution ultrasound, the serum hCG level of discriminatory zone has declined from $6,500 \mathrm{mIU} / \mathrm{ml}$ in transabdominal ultrasound (35) to 1,000 to $2,000 \mathrm{mIU} / \mathrm{ml}$ when approached with transvaginal ultrasound (36-38). However, Connolly (39), raised concern that currently used discriminatory serum hCG levels might be too low for use in clinical practice and may result in early offer for management that causes interrupted viable gestations. Moreover, an initial hCG level of $50 \%$ of EP in PUL was below the discrimination threshold (40). Up until recently, ideas of discriminatory zone have been varied, and single measurements were believed to limit the evaluation of PUL (41). Our study found that the proportion of patients with $\mathrm{EP}$ whose serum hCG $<1,000 \mathrm{mIU} / \mathrm{ml}$ was less than those with non-EP (29.1 vs. $70.9 \%$ ), unlike the prospective study by Malek-Mellouli et al., which found that 2.5\% of EP patients have serum hCG level $<1,000 \mathrm{mIU} / \mathrm{ml}$ (18). Mol et al. (42) proposed the concept of serum hCG measurement interpretation with transvaginal ultrasound findings, using hCG cutoff $\geq 1,500$ $\mathrm{mIU} / \mathrm{ml}$ in addition to positive findings in ultrasound (complex adnexal mass or fluid in cul-de-sac) (42). Surprisingly, our results found the cutoff using $\geq 1,000 \mathrm{mIU} / \mathrm{ml}$ as significantly relevant to $\mathrm{EP}$.

Therefore, the concept of all earlier mentioned factors associated with EP proposed the idea of making diagnosis as multifactorial scores. Many published predictive models were developed. Malek-Mellouli et al. proposed the logistic regression model based on three factors including serum progesterone level, bleeding per vagina with abdominal pain, and free fluid from ultrasound finding. It resulted in sensitivity of 0.79 (95\% $\mathrm{CI}=0.62-0.91)$ and specificity of $0.59(95 \% \mathrm{CI}=0.42-$ 0.73). Unfortunately, when each one factor was compared for its prediction separately, there was no significant difference in EP prediction (18). In the latter study, Condous et al. $(7,43)$ developed two predictive models, which was M1 in 2004 and M4 in 2007. Analysis performed by receiver operator characteristic curve has a good prediction with sensitivity of $73.3 \%$ and specificity of $87.3 \%$ for the M1 model. Note that the positive predictive value in M1 was only $27.5 \%$. In addition, the M4 model, using serial serum hCG at 0 and $48 \mathrm{~h}$, has a better outcome in terms of sensitivity (80\%) and specificity ( $88.6 \%)$, but there was a limitation, as longer duration of serum hCG has to be followed up. Thus, diagnosis could be delayed. Moreover, in 2010, Barnhart K. et al. (8) attempted to validate M4 model studied from the United Kingdom with a new study population in the United States. Interestingly, they found decreasing sensitivity and specificity in US population (54.8 and 87.7\%) when comparing with the population in the United Kingdom, where the model was originally developed (80 and $88.6 \%$ ), respectively. Gevaert et al. (44) introduced Bayesian network prediction using a more complex calculation with probabilistic model based on GA, serum hCG ratio, and serum progesterone level. However, according to its sensitivity of $77 \%$ and specificity of $83 \%$, the model has never been tested for its validation. In our study, the predictive models including the of five parameters achieved an accuracy of $77.8 \%$, a specificity of $91 \%$, and sensitivity of $67 \%$. 
So far, we believe that the development of a predictive model requires two important basic concepts, which are discrimination and calibration.

Our study achieves the objective of developing models for EP diagnosis among patients with symptomatic pregnancy whose first visit evaluation was PUL. Interestingly, we created the predictive scoring model that not only seem to exhibit good performance and reproducibility but was also suitable for practical use. The main goal was to provide an alternative tool for physicians dealing with PUL deciding whether intervention is necessary when $\mathrm{EP}$ was suspected before the rupture happens.

In a cross-sectional retrospective study, we encountered the inevitable limitation of missing values. As a result, we designed our study based on three separated domains (clinical, serum marker, and ultrasound), from which in turn we could collect and analyze each individual domain separately, thus preserving more data. In addition, the study ran over a period of 10 years; variation in defining terms for PUL could have changed overtime, as well as the treatment policy. Moreover, ultrasonographers' experiences and equipment details (environment of equipment and serum hCG interpretation equipment) may also be the causes for variation in this study. However, our present study developed in the single-center setting with adequate power for interpretation could help to minimize the confounding factor caused by differences in management of PUL patients, thus improving the consistency of the result. However, our dataset was obtained from a single medical unit, which may limit the generalization of the model. Consequently, our future work will aim not only to enhance the model internal validity with new datasets, but we also plan to generalize the model with other units. Thus, a multicenter prospective study would be planned for model validation.

\section{CONCLUSION}

Our study proposed a new analytic predictive model for EP, based on a risk scoring system, revealing five potent factors that could markedly improve the basic knowledge of the disease. As far as we know, it is the first analytic model in Asian populations;

\section{REFERENCES}

1. Drife J, Lewis G. Why Mothers Die 2000-2002 - The Sixth Report of Confidential Enquiries into Maternal Deaths in the United Kingdom. London: Royal College of Obstetricians and Gynaecologists (2004).

2. Kaplan BC, Dart RG, Moskos M, Kuligowska E, Chun B, Adel Hamid M, et al. Ectopic pregnancy: prospective study with improved diagnostic accuracy. Ann Emerg Med. (1996) 28:10-7. doi: 10.1016/S0196-0644(96)70131-2

3. Tay JI, Moore J, Walker JJ. Ectopic pregnancy. West J Med. (2000) 173:1314. doi: 10.1136/ewjm.173.2.131

4. Kirk E, Daemen A, Papageorghiou AT, Bottomley C, Condous G, De Moor $\mathrm{B}$, et al. Why are some ectopic pregnancies characterized as pregnancies of unknown location at the initial transvaginal ultrasound examination?. Acta Obstet Gynecol Scand. (2008) 87:1150-4. doi: 10.1080/00016340802443822

5. Barnhart K, Sammel MD, Chung K, Zhou L, Hummel AC, Guo W. Decline of serum human chorionic gonadotropin and spontaneous complete abortion: defining the normal curve. Obstet Gynecol. (2004) 104:97581. doi: 10.1097/01.AOG.0000142712.80407.fd regarding high accuracy and specificity, we hope this could be a tool for clinical decision making toward efficient management for EP patients. Thus, we hope that this research opens the way for diagnostic tool creation for other life-threatening diseases. Further study is required to validate the model with different patient cohorts.

\section{DATA AVAILABILITY STATEMENT}

The raw data supporting the conclusions of this article will be made available by the authors, without undue reservation.

\section{ETHICS STATEMENT}

The studies involving human participants were reviewed and approved by Royal Thai Army Medical Department Institutional Review Board. Written informed consent for participation was not required for this study in accordance with the national legislation and the institutional requirements.

\section{AUTHOR CONTRIBUTIONS}

PR collected the data and performed data analysis. Both authors contributed to the conception and design of the study. Both authors interpreted data, drafted, and revised the manuscript as well as approved the version of the manuscript to be published.

\section{FUNDING}

This study was supported by Phramongkutklao Research Fund.

\section{ACKNOWLEDGMENTS}

The authors express their grateful thanks to KR: contributed to study design and hypothesis, data interpretation, and drafting and revisions of the manuscript. Lastly, Department of obstetrics and gynecology, Phramongkutklao College of Medicine, for their support in completing this study. The authors wish to thank Mr. Thomas Mc Manamon for proofreading the manuscripts.
6. Bickell NA, Bodian C, Anderson RM, Kase N. Time and risk of ruptured tubal pregnancy. Obstet Gynecol. (2004) 104: 789-94. doi: 10.1097/01.AOG.0000139912.65553.58

7. Condous G, Okaro E, Khalid A, Timmerman D, Lu C, Zhou Y, et al. The use of a new logistic regression model for predicting the outcome of pregnancies of unknown location. Hum Reprod. (2004) 19:190010. doi: 10.1093/humrep/deh341

8. Barnhart K, Sammel M, Appleby D, Rausch M, Molinaro T, Van Calster B, et al. Does a prediction model for pregnancy of unknown location developed in the UK validate on a US population? Hum Reprod. (2010) 25:243440. doi: 10.1093/humrep/deq217

9. Weström L, Joesoef R, Reynolds G, Hagdu A, Thompson SE. Pelvic inflammatory disease and fertility. A cohort study of 1,844 women with laparoscopically verified disease and 657 control women with normal laparoscopic results. Sex Transm Dis. (1992) 19:185-92. doi: 10.1097/00007435-199207000-00001

10. Westrom L. Effect of pelvic inflammatory disease on fertility. Venereology. (1995) 8:219-22. 
11. Berenson A, Hammill H, Martens M, Faro S. Bacteriologic findings with ectopic pregnancy. J Reprod Med. (1991) 36:118-20.

12. Li C, Zhao WH, Zhu Q, Cao SJ, Ping H, Xi X, et al. Risk factors for ectopic pregnancy: a multi-center case-control study. BMC Pregnancy Childbirth. (2015) 15:187. doi: 10.1186/s12884-015-0613-1

13. Mehta HB, Mehta V, Girman CJ, Adhikari D, Johnson ML. Regression coefficient-based scoring system should be used to assign weights to the risk index. J Clin Epidemiol. (2016) 79:22-8. doi: 10.1016/j.jclinepi.2016. 03.031

14. Condous G, Kirk E, Van Calster B, Van Huffel S, Timmerman D, Bourne T. Failing pregnancies of unknown location: a prospective evaluation of the human chorionic gonadotrophin ratio. Bjog. (2006) 113:5217. doi: 10.1111/j.1471-0528.2006.00924.x

15. Hahlin M, Thorburn J, Bryman I. The expectant management of early pregnancies of uncertain site. Hum Reprod. (1995) 10:1223-7. doi: 10.1093/oxfordjournals.humrep.a136123

16. Banerjee S, Aslam N, Zosmer N, Woelfer B, Jurkovic D. The expectant management of women with early pregnancy of unknown location. Ultrasound Obstet Gynecol. (1999) 14:2316. doi: 10.1046/j.1469-0705.1999.14040231.x

17. Banerjee S, Aslam N, Woelfer B, Lawrence A, Elson J, Jurkovic D. Expectant management of early pregnancies of unknown location: a prospective evaluation of methods to predict spontaneous resolution of pregnancy. Bjog. (2001) 108:158-63. doi: 10.1111/j.1471-0528.2001.00031.x

18. Malek-Mellouli M, Oumara M, Ben Amara F, Zouch O, Neji K, Reziga H. Prediction of ectopic pregnancy in early pregnancy of unknown location. Tunis Med. (2013) 91:27-32.

19. Kirk E, Condous G, Van Calster B, Van Huffel S, Timmerman D, Bourne T. Rationalizing the follow-up of pregnancies of unknown location. Hum Reprod. (2007) 22:1744-50. doi: 10.1093/humrep/dem073

20. ACOG practice bulletin No. 193: tubal ectopic pregnancy. Obstet Gynecol. (2018) 131:e91-103. doi: 10.1097/AOG.0000000000002560

21. Essa AA, Madny EH, Zayed OM, Elshaboury IM, Mahmoud BM. Evaluation of prognostic value of Fernandez scoring system on the management of ectopic pregnancy in the emergency room at Suez Canal University Hospitals, Egypt. Int Surg J. (2017) 4:1292-8. doi: 10.18203/2349-2902.isj20171130

22. Bouyer J, Coste J, Shojaei T, Pouly J-L, Fernandez H, Gerbaud L, et al. Risk factors for ectopic pregnancy: a comprehensive analysis based on a large case-control, population-based study in France. Am J Epidemiol. (2003) 157:185-94. doi: 10.1093/aje/kwf190

23. Saxon D, Falcone T, Mascha EJ, Marino T, Yao M, Tulandi T. A study of ruptured tubal ectopic pregnancy. Obstet Gynecol. (1997) 90:469. doi: 10.1016/S0029-7844(97)00180-4

24. Ammon Avalos L, Galindo C, Li DK. A systematic review to calculate background miscarriage rates using life table analysis. Birth Defects Res A Clin Mol Teratol. (2012) 94:417-23. doi: 10.1002/bdra.23014

25. Shaw JL, Dey SK, Critchley HO, Horne AW. Current knowledge of the aetiology of human tubal ectopic pregnancy. Hum Reprod Update. (2010) 16:432-44. doi: 10.1093/humupd/dmp057

26. Berek, Jonathan S. Berek \& Novak's Gynecology. 16th ed. Philadelphia, PA: Wolters Kluwer Health/Lippincott Williams \& Wilkins (2019).

27. Shurie S, Were E, Orang'o O, Keter A. Levonorgestrel only emergency contraceptive use and risk of ectopic pregnancy in Eldoret Kenya: a case-control study. Pan Afr Med J. (2018) 31:214. doi: 10.11604/pamj.2018.31.214.17484

28. Cleland K, Raymond E, Trussell J, Cheng L, Zhu H. Ectopic pregnancy and emergency contraceptive pills: a systematic review. Obstetr Gynecol. (2010) 115:1263-6. doi: 10.1097/AOG.0b013e3181dd22ef

29. Moini A, Hosseini R, Jahangiri N, Shiva M, Akhoond MR. Risk factors for ectopic pregnancy: a case-control study. J Res Med Sci. (2014) 19:844-9.

30. Coste J, Job-Spira N, Fernandez H. Increased risk of ectopic pregnancy with maternal cigarette smoking. Am J Public Health. (1991) 81:199201. doi: 10.2105/AJPH.81.2.199
31. Buckley RG, King KJ, Disney JD, Gorman JD, Klausen JH. History and physical examination to estimate the risk of ectopic pregnancy: validation of a clinical prediction model. Ann Emerg Med. (1999) 34:58994. doi: 10.1016/S0196-0644(99)70160-5

32. Kirk E, Papageorghiou AT, Condous G, Tan L, Bora S, Bourne T. The diagnostic effectiveness of an initial transvaginal scan in detecting ectopic pregnancy. Hum Reprod. (2007) 22:2824-8. doi: 10.1093/humrep/ $\operatorname{dem} 283$

33. Richardson A, Gallos I, Dobson S, Campbell B, Coomarasamy A, RaineFenning N. Accuracy of first-trimester ultrasound in diagnosis of tubal ectopic pregnancy in the absence of an obvious extrauterine embryo: systematic review and meta-analysis. Ultrasound Obstetr Gynecol. (2016) 47:28-37. doi: 10.1002/uog.14844

34. Murray H, Baakdah H, Bardell T, Tulandi T. Diagnosis and treatment of ectopic pregnancy. Cmaj. (2005) 173:905-12. doi: 10.1503/cmaj.050222

35. Kadar N, DeVore G, Romero R. Discriminatory hCG zone: its use in the sonographic evaluation for ectopic pregnancy. Obstetr Gynecol. (1981) 58:156-61.

36. Cacciatore B, Stenman UH, Ylöstalo P. Diagnosis of ectopic pregnancy by vaginal ultrasonography in combination with a discriminatory serum hCG level of 1000 IU/1 (IRP). BJOG: Int J Obstetr Gynaecol. (1990) 97:9048. doi: 10.1111/j.1471-0528.1990.tb02445.x

37. Barnhart K, Mennuti MT, Benjamin I, Jacobson S, Goodman D, Coutifaris C. Prompt diagnosis of ectopic pregnancy in an emergency department setting. Obstetr Gynecol. (1994) 84:1010-5.

38. Barnhart KT, Simhan H, Kamelle SA. Diagnostic accuracy of ultrasound above and below the beta-hCG discriminatory zone. Obstetr Gynecol. (1999) 94:583-7. doi: 10.1097/00006250-199910000-00018

39. Connolly A, Ryan DH, Stuebe AM, Wolfe HM. Reevaluation of discriminatory and threshold levels for serum $\beta$-hCG in early pregnancy. Obstetr Gynecol. (2013) 121:65-70. doi: 10.1097/AOG.0b013e318278f421

40. Kirk E, Condous G, Bourne T. Pregnancies of unknown location. Best Pract Res Clin Obstetr Gynaecol. (2009) 23:4939. doi: 10.1016/j.bpobgyn.2009.01.008

41. Cartwright PS, Moore RA, Dao AH, Wong SW, Anderson JR. Serum beta-human chorionic gonadotropin levels relate poorly with the size of a tubal pregnancy. Fertil Steril. (1987) 48:679-80. doi: 10.1016/S0015-0282(16)59485-0

42. Mol BW, Hajenius PJ, Engelsbel S, Ankum WM, Van der Veen F, Hemrika DJ, et al. Serum human chorionic gonadotropin measurement in the diagnosis of ectopic pregnancy when transvaginal sonography is inconclusive. Fertil Steril. (1998) 70:972-81. doi: 10.1016/S0015-0282(98) 00278-7

43. Condous G, Van Calster B, Kirk E, Haider Z, Timmerman D, Van Huffel $S$, et al. Prediction of ectopic pregnancy in women with a pregnancy of unknown location. Ultrasound Obstetr Gynecol. (2007) 29:680-7. doi: 10.1002/ uog. 4015

44. Gevaert O, De Smet F, Kirk E, Van Calster B, Bourne T, Van Huffel S, et al. Predicting the outcome of pregnancies of unknown location: Bayesian networks with expert prior information compared to logistic regression. Hum Reprod. (2006) 21:1824-31. doi: 10.1093/humrep/del083

Conflict of Interest: The authors declare that the research was conducted in the absence of any commercial or financial relationships that could be construed as a potential conflict of interest.

Copyright (c) 2021 Rueangket and Rittiluechai. This is an open-access article distributed under the terms of the Creative Commons Attribution License (CC BY). The use, distribution or reproduction in other forums is permitted, provided the original author(s) and the copyright owner(s) are credited and that the original publication in this journal is cited, in accordance with accepted academic practice. No use, distribution or reproduction is permitted which does not comply with these terms. 